consideration of EVT. Of those transferred, 130 (59.9\%) underwent EVT at the CSC. Transferred patients were more likely to receive t-PA $(24.4 . \%$ vs $10.9 \%)$ and have an anterior circulation LVO $(86.0 \%$ vs $63.0 \%)$ than patients not transferred. Median distance to CSC for patients transferred from PSC was not significantly different to patients not transferred (21 miles vs 20 miles).

Conclusions LVO patients who presented to PSCs were less likely to receive EVT than patients directly presenting to a CSC. Anterior circulation LVOs were more likely to be transferred for EVT evaluation. Despite a higher NIHSS, and similar other baseline characteristics, female patients were less likely to be routed to a CSC.

Disclosures M. Tariq: None. S. Salazar-Marioni: None. S. Khose: None. L. Mccullough: 1; C; American Heart Association, National Institute of Health. V. Lopez: None. R. AbdelKhaleq: None. Y. Kim: None. S. Sheth: None.

\section{0-035 HYPERACUTE INFLAMMATORY PROFILE IN PATIENTS WITH ACUTE ISCHEMIC STROKE (AIS)}

M Farooqui, C Zevallos, D Quispe-Orozco, A Dajles, A Mendez Ruiz, D Tranel, N Karandikar, S Ortega, S Ortega-Gutierrez*. University of lowa Hospitals and Clinics, lowa City, IA

\subsection{6/neurintsurg-2021-SNIS.35}

Introduction Inflammation is an important mechanism of ischemic brain injury. It is characterized by inflammatory mediators and molecules including cytokines and interleukins. This inflammatory mechanism and its interaction among these Ischemic Stroke (IS) patients is still ambiguous. The aim of this investigation is to elucidate and characterize the early inflammatory response at the site of occlusion among these IS patients.

Methods Large vessel occlusion acute ischemic stroke (LVOAIS) patients eligible for Mechanical Thrombectomy (MT) were recruited within 24 hours from their symptom onset. Blood samples were collected proximal and distal to the occlusion site during the procedure. Control samples were collected from the femoral artery and median cubital vein. 20-Plex assay and ELISA was used for the analysis of cytokines and chemokines. Graph-pad prism and R-software was used for evaluating the differences among the molecules across the site of occlusion and control.

Results A total of 19 (male: 13 and female: 6) patients were included. Cytokine quantification observed a significant increase in MMP-9 and IFN-g proximal to the occlusion, whereas, there was a decrease in IL-2, IL-4, IL-5, IL-6, IL-7, IL-15，IL-17，GM-CSF，TNF- $\alpha$, IP-10, VEGF, MIP-1a, and MIP-1b distal to the clot. The levels of IL-8, MCP-1, and MIG were comparable across the sites.

Conclusion Our results characterized the local environment and immediate inflammatory molecules, within few hours of the ischemic brain injury. These observations indicate the evidence of initial activation of inflammatory response. This will help better understand the molecular patho-physiology and identify molecular biomarkers of ischemic stroke progression and subsequent modulating therapeutic interventions.

Disclosures M. Farooqui: None. C. Zevallos: None. D. Quispe-Orozco: None. A. Dajles: None. A. Mendez Ruiz: None. D. Tranel: None. N. Karandikar: None. S. Ortega: None. S. Ortega-Gutierrez: None.

\section{0-036 DIRECT PUNCTURE OF THE SUPERIOR OPHTHALMIC VEIN FOR CAROTID CAVERNOUS FISTULAS: A 20-YEAR EXPERIENCE AND REVIEW OF LITERATURE}

1J Catapano*, ${ }^{1} \mathrm{R}$ Singh, ${ }^{1} \mathrm{~N}$ De la Pena, ${ }^{1} \mathrm{D}$ Wilkinson, ${ }^{1} \mathrm{~J}$ Baranoski, ${ }^{1} \mathrm{~V}$ Srinivasan, ${ }^{1} \mathrm{C}$ Rutledge, ${ }^{2} \mathrm{~A}$ Jadhav, ${ }^{1} \mathrm{~A}$ Ducruet, ${ }^{1} \mathrm{~F}$ Albuquerque. 'Neurosurgery, BNI, Phoenix, AZ; ${ }^{2}$ Neurosurgery, Barrow, Phoenix, AZ

\subsection{6/neurintsurg-2021-SNIS.36}

Introduction Carotid Cavernous Fistulas (CCF) can be challenging and may require unconventional approaches for treatment. The superior ophthalmic vein (SOV) is an alternative approach for embolization of CCFs. However, only a few case series have been published on the direct cannulation of the SOV for CCFs. The present study analyses the single largest series to date of direct SOV puncture for treatment of CCFs, as well as a review of literature.

Methods All patients treated for a CCF with direct SOV cannulation from $1 / 1 / 2000$ to $12 / 31 / 2020$ were retrospectively analyzed at a single quaternary center. Patient demographic, characteristics, and outcomes were analyzed. An additional review of literature for all case series for direct puncture of the SOV for CCF treatment was performed.

Results During the 20-year study period, direct cannulation of the SOV for treatment of a CCF was attempted on 17 patients, with one patient $(6 \%)$ having the procedure aborted due to inability to navigate the wire. In the 16 patients with direct SOV CCF treatment, the average age was 54 (sD 21.9) and 8 (50\%) were female. The most common

\begin{tabular}{|c|c|c|c|c|c|c|c|}
\hline CASE ID & AGE & SEX & SIGNS/Symptoms & Laterallzation & Patholozy & Calse & Prior Falled Procedures \\
\hline 1 & 84 & Female & Diploplia (VI palsy), Ptosis, Periorbital Swelling & Right & Indirect CCF & Trauma & Yes \\
\hline 2 & 59 & Female & Diploplia (VI palsy), Periorbital Swelling,Ophthalmoplegia , Headache, Chemosis & Left & Indirect CCF & Unknown & Yes \\
\hline 3 & 51 & Female & Diplopia (N palsy), Visual Loss, Proptosis, Chemosis & Left & Indirect $\mathrm{CCF}$ & Unknown & Yes \\
\hline 4 & 75 & Male & Diplopia (IV palsy), Proptosis, Pain, Chemosis & Left & Indirect CCF & Unknown & Yes \\
\hline 5 & 35 & Male & Visual Loss, Pain, Periorbital Swelling, Chemosis, Diplopia (IV palsy), Ptosis & Right & Indirect CCF & Trauma & Yes \\
\hline 6 & 60 & Female & Chemosis, Proptosis, Periorbital swelling, Blurriness, Diplopia (IV palsy) & Left & Indirect $\mathrm{CCF}$ & Uniknown & Yes \\
\hline 7 & 48 & Male & Headache, Diplopia (VI), Ophthalmoplegia, Periorbital swelling, Visual Loss, Pain & Right & Indirect $\mathrm{CCF}$ & Unknown & Yes \\
\hline 8 & 71 & Female & Visual loss, Proptosis, Chemosis, Ophthaimoplegia & Right & Indirect CCF & Unknown & Yes \\
\hline 9 & 80 & Female & Chemosis, Proptosis, Diplopia (VI palsy) & Left & Indirect CCF & Trauma & Yes \\
\hline 10 & 57 & Male & Headache, Diplopia (VI), Chemosis & Left & Indirect CCF & Trauma & Yes \\
\hline 11 & 20 & Male & Bruit, Chemosis, Proptosis & Left & Indirect CCF & Trauma & Yes \\
\hline 12 & 79 & Female & Pain, Periorital Edema, Opthalmoplegia, Ptosis & Right & Indirect $O C F$ & Unknown & Yes \\
\hline 13 & 43 & Male & Chemosis, Proptosis, Visual Loss & Left & Indirect CCF & Unknown & Yes \\
\hline 14 & 49 & Female & Headache, Visual Loss, Diplopia (IV palsy) & Left & Indirect CCF & Unknown & Yes \\
\hline 15 & 74 & Female & Periorbital Edema, Pain, Tinnitus, Diplopia (VI palsy), Visual Loss & Right & Indirect $\mathrm{CCF}$ & Trauma & Yes \\
\hline 16 & 27 & Male & Chemosis, Visual Loss, Diplopia [IV palsy] & Left & Indirect CCF & Trauma & Yes \\
\hline 17 & 17 & Male & Bruit, Ptosis, Tinnitus, Visual Loss, Diplopia (VI palsy) & Left & Indirect CCF & Trauma & Yes \\
\hline
\end{tabular}

